\title{
Correlative HAADF-STEM and EDX-STEM Tomography for the 3D Morphological and Elemental Analysis of FinFET Semiconductor Devices
}

\author{
Julien Sorel ${ }^{1,2}$, Martin Jacob ${ }^{1}$, Toby Sanders ${ }^{3}$, Adeline Grenier ${ }^{1}$, Rafael Bortolin Pinheiro ${ }^{1}$, Frédéric \\ Mazen $^{1}$, Thierry Epicier ${ }^{2}$ and Zineb Saghi ${ }^{1}$ \\ 1. Univ. Grenoble Alpes, CEA, LETI, Grenoble, France. \\ 2. Univ Lyon, INSA-Lyon, Université C. Bernard Lyon 1, Villeurbanne Cedex, France. \\ 3. School of Mathematical and Statistical Sciences, Arizona State University, Tempe, AZ.
}

The increasing complexity of semiconductor devices demands advanced three-dimensional (3D) characterization techniques for elucidation of structure-property relationships and optimization of process conditions. High angle annular dark field scanning transmission electron microscope (HAADFSTEM) tomography has greatly contributed to the 3D morphological and structural understanding of crystalline materials at the nanoscale [1]. 3D chemical analysis with energy-dispersive Xray spectroscopy STEM (EDX-STEM) tomography is nowadays possible thanks to the recent developments in electron sources and X-ray detectors with improved efficiency [2]. Combining both techniques is a promising route to provide complementary insights into the characterization of nanodevices. In this work, we applied both techniques to a silicon fin-shaped structure implanted with Arsenic (As) as n-type dopant and encapsulated in silicon oxide $\left(\mathrm{SiO}_{2}\right)$. A needle-shaped sample was prepared by focused ion beam (FIB) and mounted onto a Fischione on-axis holder. The acquisitions were performed using an FEI Titan Themis operating at $200 \mathrm{kV}$ and equipped with a probe corrector and 4 SDD EDX detectors: an HAADF-STEM tilt series was acquired between $-90^{\circ}$ and $+90^{\circ}$ with $5^{\circ}$ increment. The frame size was 2048x2048 pixels, with a frame time of 15 seconds and a pixel size of $0.26 \mathrm{~nm}$. In parallel, every $10^{\circ}$, an EDX-STEM map was acquired using Esprit software (Bruker). In this case, a frame size of 193x163 pixels was chosen, with an acquisition time of $10 \mathrm{~min} / \mathrm{map}$ and a pixel size of $1 \mathrm{~nm}$. Hyperspy, a python-based software for hyperspectral data processing [3], was then used for the batch-processing of the 4D dataset. Spectral unmixing using non-negative matrix factorization (NMF) led to the identification of three chemical phases in the sample: $\mathrm{Si}, \mathrm{SiO}_{2}$ and As, as can be seen on Figure 1(b-d) (the corresponding HAADF-STEM image is shown in Figure 1(a)). The extracted tilt series, along with the HAADF-STEM dataset, were aligned using the common line and mass center methods, and the reconstructions were performed using the total variation minimization algorithm (TVM), necessary for such under-sampled datasets [4]. Figure 2(a) and (b) show the volume rendering and a slice through the HAADF-STEM reconstruction, where the roughness of the Si nanowire is well resolved, and can be reliably quantified in 3D using for instance the solidity measure (Volume/convex volume). The average volume of the As precipitates was estimated to $\sim 15 \mathrm{~nm}^{3}$. The reliability of this measure depends on the accuracy of the segmentation step (and the robustness of the reconstruction algorithm). EDX-STEM tomography led to an As volume (Figure 2(d) and (f)) which was easier to segment, but with a worse resolution than HAADF-STEM, due to large pixel size and tilt increment. The average volume of the As precipitates in this case was estimated to $\sim 20 \mathrm{~nm}^{3}$. EDX-STEM tomography however provided information about the 3D implantation of As that was not present in the HAADF-STEM volume. As shown in Figure 2(g), a depth profile though the fin shows an implantation of approximatively $20 \mathrm{~nm}$. 
In summary, it is shown here that HAADF-STEM tomography remains very useful in parallel to EDXbased 3D analysis of complex structures, the coupling of both approaches leading to a thorough and very complementary information.

\section{References:}

[1] C. Kübel et al, Microsc. Microanal. 111 (2005), p. 378.

[2] K. Lepinay et al, Micron 47 (2013), p. 43.

[3] F. De La Peña et al, Hyperspy 1.3, ed. Zenodo.

[4] T. Sanders et al, Adv. Struct. Chem. Imaging 1 (2015).
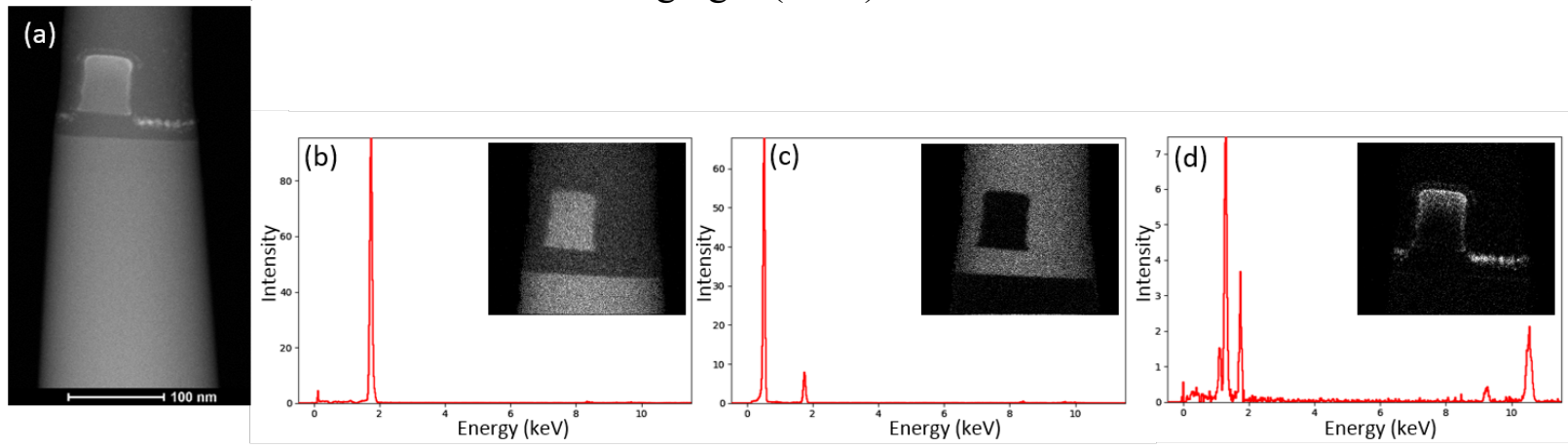

Figure 1. (a) HAADF-STEM projection of silicon fin-shaped structure at $-5^{\circ}$. (b-d) NMF unmixing applied to the EDX-STEM tomographic dataset led to the identification of 3 chemical phases: $\mathrm{Si}, \mathrm{SiO}_{2}$ and As.
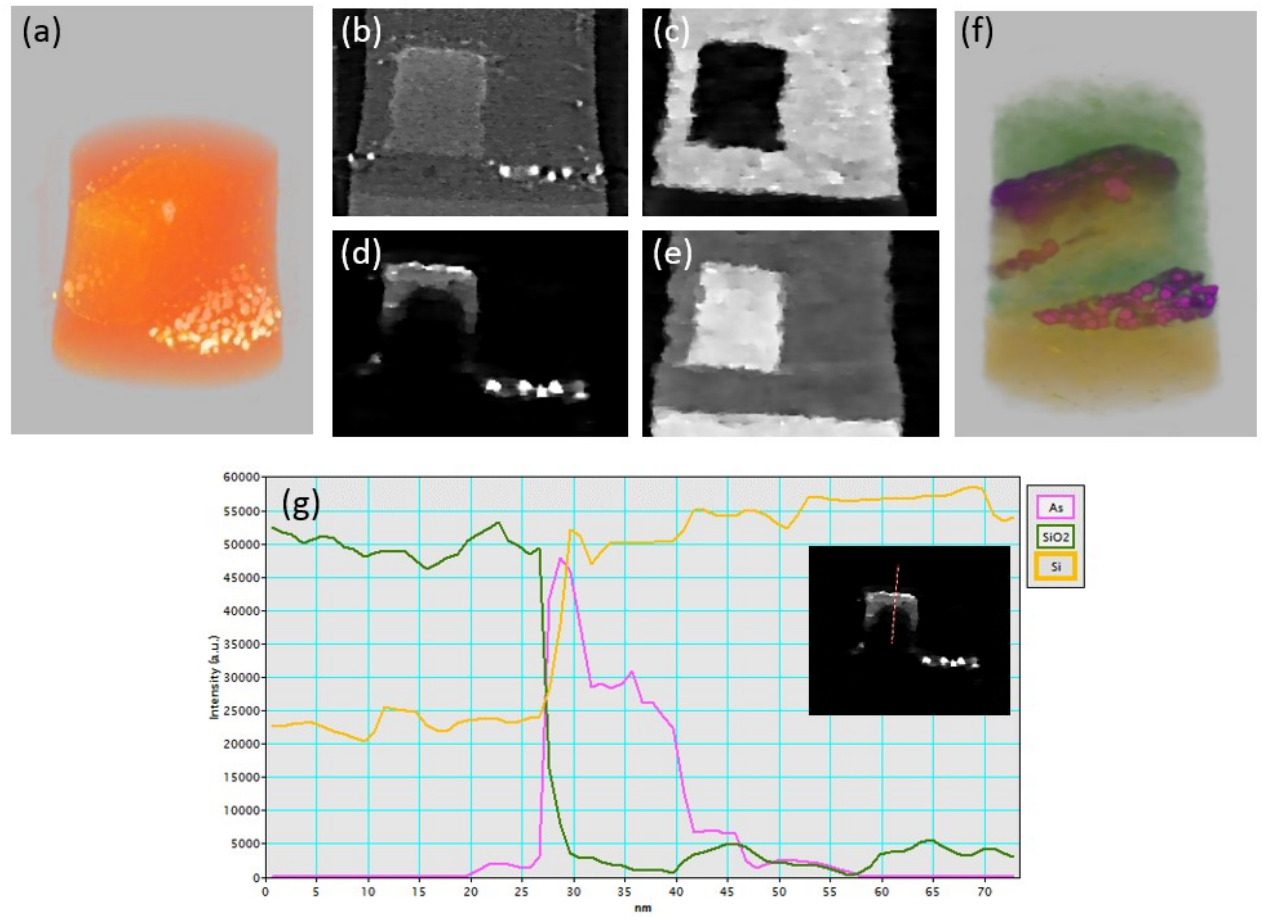

Figure 2. (a) Volume rendering and (b) a slice through the HAADF-STEM tomographic reconstruction. (c-e) are slices through the $\mathrm{SiO}_{2}$, As, and Si reconstructions obtained with EDX-STEM tomography. (f) Volume rendering of the tomographic reconstructions of $\mathrm{SiO}_{2}$ (green), As (purple) and $\mathrm{Si}$ (yellow) chemical phases. (g) A depth profile through the fin on a selected section (dotted line in the insert). 